\title{
Connections between Wiener index and matchings
}

\author{
Weigen Yan*,*** \\ School of Sciences, Jimei University, Xiamen 361021, China \\ Institute of Mathematics, Academia Sinica, Taipei 11529, Taiwan \\ E-mail: weigenyan@263.net \\ Yeong-Nan Yeh** \\ Institute of Mathematics, Academia Sinica, Taipei 11529, Taiwan \\ E-mail: mayeh@math.sinica.edu.tw.
}

Received 15 August 2005; revised 17 August 2005

Let $T$ be an acyclic molecule with $n$ vertices, and let $S(T)$ be the acyclic molecule obtained from $T$ by replacing each edge of $T$ by a path of length two. In this work, we show that the Wiener index of $T$ can be explained as the number of matchings with $n-2$ edges in $S(T)$. Furthermore, some related results are also obtained.

KEY WORDS: Wiener index, matching, perfect matching, Hosoya index, Laplacian matrix

MSC: $05 \mathrm{C} 12$

\section{Introduction}

The study of various molecular-graph-based structure descriptors, so-called "topological indices" (see, for example, [1-3]), has been undergoing rapid expansion in the last few years. Without question, the Wiener index is one of the best known and most examined among them. The Wiener index of an acyclic molecule $T$ with the vertex set $V(T)$, denoted by $W(T)$, is defined as

$$
W(T)=\sum_{\{u, v\} \subseteq V(T)} d_{T}(u, v),
$$

where $d_{T}(u, v)$ is the distance between vertices $u$ and $v$ in $T$.

*This work is supported by FMSTF (2004J024) and NSFF(E0540007).

** Partially supported by NSC94-2115-M001-017.

*** Corresponding author. 
The name Wiener index or Wiener number for the quantity defined in (1) is usual in chemical literature, since Wiener [4] in 1947 seems to be the first person that considered it. Wiener defined it in a slightly different yet equivalent way. $\mathrm{He}$ used it to describe the variations in the number of physicochemical properties of alkanes [4-8]; the definition of the Wiener index in terms of distances between vertices of a graph, such as in (1), was the first given by Hosoya [9].

Suppose $u$ is a vertex of $T$. The partial Wiener index was introduced in [1012] as follows:

$$
W(T, u)=\sum_{v \in V(T)} d_{T}(u, v) .
$$

It is well known [9] that the formula (1) is equivalent to the following formula:

$$
W(T)=\sum_{e} n_{1}(e) n_{2}(e),
$$

where $e=(u, v)$ is an edge of $T$, and $n_{1}(e)$ (resp. $n_{2}(e)$ ) is the number of vertices of $T$ lying closer to $u$ than to $v$ (resp. lying closer to $v$ than to $u$ ), and the summation is over all edges of $T$.

Based on the formula (3), it is natural to generalize the definition of $W(T)$ as follows.

Let $U$ be a subset of the edge set $E(T)$ of an acyclic molecule $T$, and let $T-U$ be the acyclic graph obtained from $T$ by deleting all edges in $U$. Obviously, if $|U|=i$ then $T-U$ contains exactly $i+1$ components, denoted by $U\left(T_{1}\right), U\left(T_{2}\right), \ldots, U\left(T_{i+1}\right)$. Denote the number of vertices of $U\left(T_{j}\right)$ by $n_{j}(U)$ for $j=1,2, \ldots, i+1$. Define the generalized Wiener indices $W_{i}(T)$ as

$$
W_{i}(T)=\sum_{U \subseteq E(T),|U|=i} n_{1}(U) n_{2}(U) \cdots n_{i+1}(U)
$$

for $i=0,1,2, \ldots, n-1$, where the summation ranges over all subsets of $E(T)$ with $i$ elements.

Obviously, $W_{0}(T)=n, W_{1}(T)$ equals the Wiener index, and $W_{2}(T)$ is the modified hyper-Wiener index, which was introduced by Gutman [13]. It was demonstrated that $W_{2}(T)$ has certain advantages over the original hyper-Wiener index introduced in Randić [14].

A set $M$ of edges of an acyclic molecule $T$ with $n$ vertices is a matching if every vertex of $T$ is incident with at most one edge in $M$; it is a perfect matching if every vertex of $T$ is incident with exactly one edge in $M$. We use the notation $v \in M$ to mean that the vertex $v$ is incident with some edge in $M$, and $v \notin M$ shall 
mean that the vertex $v$ is not incident to any edge in $M$. For $j=0,1, \ldots,\lfloor n / 2\rfloor$, the weighted $j$-matching number of $T$ is defined to be

$$
m_{T}(j)=\sum_{M} \prod_{v \notin M} d(v),
$$

where the summation is over all matchings with $j$ edges of $T$ and the product ranges over all vertices which are incident with no edge in $M$, and $d(v)$ is the degree of the vertex $v$ of $T$. Denote by $m(T, i)$ the number of matchings of $G$ with $i$ edges. We set $m(T, 0)=1$ by convention. Denote by $Z(T)$ the number of matchings of $T$, that is,

$$
Z(T)=m(T, 0)+m(T, 1)+\cdots+m\left(T,\left\lfloor\frac{n}{2}\right\rfloor\right) .
$$

The number of matchings of $T$ is also called the Hosoya index (see Hosoya [9]).

The following interesting result was communicated around 1990 independently in several papers [15-18]:

$$
W(T)=n \sum_{i=1}^{n-1} \frac{1}{\lambda_{i}},
$$

where $T$ is an acyclic molecule with $n$ vertices, $\lambda_{1} \geqslant \lambda_{2} \geqslant \cdots \geqslant \lambda_{n-1}$ are the non-zero Laplacian eigenvalues of $T$.

The above result connects the Wiener index (a quantity defined on the basis of graph distances) and matrix eigenvalues. The possible chemical implications of (6) were discussed in $[19,20]$. On the other hand, by using algebraic method, Chan, Lam and Merris [21] arrived a related result, namely they expressed $W(T)$ as a linear function of a hook immanant of the Laplacian matrix of $T$. This algebraic result was used to show that $W(T)$ is formally related to other graph invariants of interest in chemistry [22]. It leads to a new interpretation of the Wiener index as a weighted sum of matchings in the molecule as follows:

$$
W(T)=\frac{1}{4}\left(\sum_{j=0}^{\lfloor n / 2\rfloor} \chi_{3}(j) m_{T}(j)+2 n(n-1)\right),
$$

where $\chi_{3}$ is an irreducible character of the symmetric group indexed by a partition $\lambda=\left(3,1^{n-3}\right)$ of $n$ and by [19] we have $\chi_{3}(j)=\frac{(-1)^{j}}{2}[(n-2 j-1)(n-2 j-2)-$ $2 j$ ], and $m_{T}(j)$ equals the weighted $j$-matchings number of $T$ defined in (5).

In addition to the formulas (1), (3), (6) and (7), many other formulas computing the Winer index of an acyclic molecule were found (see, for example, ref. $[2,3,23-26])$.

In this work, we mainly prove that if $T$ is an acyclic molecule with $n$ vertices then $W_{i}(T)$ can be expressed in terms of the number of some matchings 
of a related acyclic molecule $S(T)$ with $2 n-1$ vertices for $i=0,1,2, \ldots, n-1$. Particularly, the Wiener index $W(T)$ of $T$ equals $m(S(T), n-2)$ and the modified hyper-Wiener index $W_{2}(T)$ of $T$ equals $m(S(T), n-3)$.

\section{The subdivision graph of an acyclic molecule}

Let $T$ be an acyclic molecule with the vertex set $V(T)=\left\{v_{1}, v_{2}, \ldots, v_{n}\right\}$ and the edge set $E(T)=\left\{e_{1}, e_{2}, \ldots, e_{n-1}\right\}$. The subdivision graph $S(T)$ is defined as the acyclic molecule obtained from $T$ by replacing each edge $e=(u, v)$ of $T$ by a path $u e v$ of length two (figure 1(a) and (b) illustrate the procedure constructing $S(T)$ from $T)$. Hence the vertex set of $S(T)$ is $V(T) \cup E(T)$. The vertex set of $T$ can be naturally regarded as a subset of the vertex set of $S(T)$.

Let $B=\left(b_{i j}\right)_{n \times(n-1)}$ be the incidence matrix of $T$, where

$$
b_{i j}= \begin{cases}1 & \text { if } e_{j}=\left(v_{i}, \sim\right), \\ 0 & \text { otherwise }\end{cases}
$$

For the acyclic molecule $T$ showed in figure 1(a), we have

$$
B=\left(\begin{array}{llll}
1 & 0 & 0 & 0 \\
1 & 1 & 1 & 0 \\
0 & 1 & 0 & 0 \\
0 & 0 & 1 & 1 \\
0 & 0 & 0 & 1
\end{array}\right) .
$$

It is well known that the adjacency matrix of $S(T)$, denoted by $A(S(T))$, has the following form (see [27]):

$$
A(S(T))=\left(\begin{array}{cc}
0 & B \\
B^{T} & 0
\end{array}\right),
$$

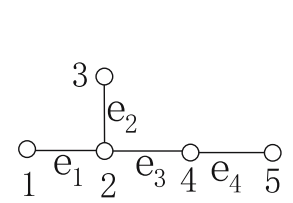

(a)

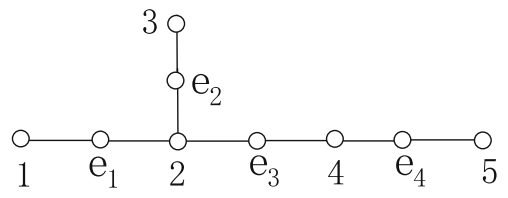

(b)

Figure 1. (a) An acyclic molecule $T$. (b) The corresponding molecule $S(T)$ of $T$. 
where $B^{T}$ is the transpose of $T$. Particularly, we have

$$
B B^{T}=D+A,
$$

where $D=\operatorname{diag}\left(d_{1}, d_{2}, \ldots, d_{n}\right)$ is the diagonal matrix of vertex degrees of $T$ and $A$ is the adjacency matrix of $T$. We call $D-A$ the Laplacian matrix of $T$. The Laplacian characteristic polynomial of $T$ is $u(T, x)=\operatorname{det}\left(x I_{n}-D+A\right)$. It can be written in the coefficient form as:

$$
\mu(T, x)=\sum_{k=0}^{n}(-1)^{k} c_{k} x^{n-k},
$$

where $c_{n}=0, c_{n-1}=n$ and $c_{0}=1$.

According to the Kel'mans theorem [20, 27, 28], the coefficients $c_{k} \mathrm{~s}$ of $\mu(T, x)$ satisfy the following:

$$
c_{n-k-1}=W_{k}(T)
$$

for $k=0,1, \ldots, n-1$.

\section{The generalized Wiener indices and matchings of $S(T)$}

Let $T$ be an acyclic molecule with $n$ vertices. Then $S(T)$ is an acyclic molecule with $2 n-1$ vertices.

Theorem 3.1. The generalized Wiener indices $W_{k}(T)$ of $T$ and the numbers of matchings of $S(T)$ with $n-k-1$ edges are equal for $k=0,1,2, \ldots, n-1$, that is,

$$
W_{k}(T)=m(S(T), n-k-1) .
$$

Particularly, the Wiener index $W(T)$ of $T$ equals $m(S(T), n-2)$ and the modified hyper-Wiener index $W_{2}(T)$ of $T$ equals $m(S(T), n-3)$.

Proof. Let $B=\left(b_{i j}\right)_{n \times(n-1)}$ be the incidence matrix of $T$. Then the adjacency matrix of $S(T)$ has the following form:

$$
A(S(T))=\left(\begin{array}{cc}
0 & B \\
B^{T} & 0
\end{array}\right) .
$$

Hence the characteristic polynomial $\phi(S(T), x)$ of $S(T)$ satisfies:

$$
\begin{aligned}
& \phi(S(T), x)=\operatorname{det}\left(x I_{2 n-1}-A(S(T))\right) \\
& \quad=\operatorname{det}\left(\begin{array}{cc}
x I_{n} & -B \\
-B^{T} & x I_{n-1}
\end{array}\right)=\frac{1}{x} \operatorname{det}\left(x^{2} I_{n}-B B^{T}\right) .
\end{aligned}
$$


By (8), we have $B B^{T}=D+A$. Hence we have

$$
\phi(S(T), x)=\frac{1}{x} \operatorname{det}\left(x^{2} I_{n}-B B^{T}\right)=\frac{1}{x} \operatorname{det}\left(x^{2} I_{n}-D-A\right) .
$$

Note that, since $T$ is an acyclic molecule, it is a bipartite graph. Hence we have (see [27])

$$
u(T, x)=\operatorname{det}\left(x I_{n}-D+A\right)=\operatorname{det}\left(x I_{n}-D-A\right) .
$$

By (11) and (12), we have

$$
\phi(S(T), x)=\frac{1}{x} u\left(T, x^{2}\right) .
$$

Since $S(T)$ is an acyclic molecule, it is well known that the characteristic polynomial $\phi(S(T), x)$ can be expressed as [27]

$$
\phi(S(T), x)=\sum_{k=0}^{n-1}(-1)^{k} m(S(T), k) x^{2 n-1-2 k},
$$

where $m(S(T), k)$ denotes the number of matchings of $S(T)$ with $k$ edges.

From (9), (10), (13) and (14), we have

$$
W_{k}(T)=m(S(T), n-k-1) .
$$

Hence the theorem has been proved.

From the above result, we have the following:

Corollary 3.1. Let $T$ be an arbitrary acyclic molecule with $n$ vertices. Then the number of matchings of $S(T)$ with $n-1$ edges is $n$, which is independent of the structure of $T$.

Corollary 3.2. Let $T$ be an arbitrary acyclic molecule with $n$ vertices. Then the Hosoya index of $S(T)$ equals $(-1)^{n} u(T,-1)$, that is,

$$
Z(S(T))=(-1)^{n} u(T,-1)=\sum_{k=0}^{n-1} W_{k}(T) .
$$

Now we consider the relation between the partial Winer index of an acyclic molecule and the matchings of $S(T)$.

Lemma 3.1. If $T$ is an acyclic molecule with $n \geqslant 2$ vertices, then, for an arbitrary vertex $v$ of $T, S(T)-v$ contains a perfect matching, where $S(T)-v$ is the graph obtained from $S(T)$ by deleting $v$ and the incident edges. 


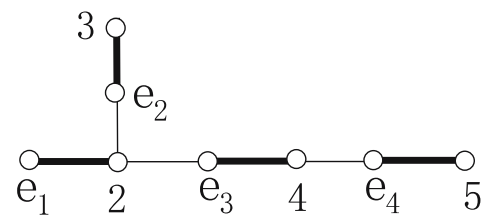

(a)

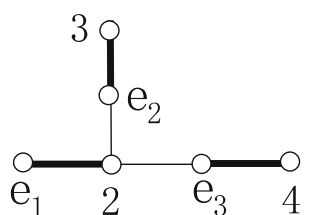

(b)

Figure 2. (a) The acyclic molecule $S(T)-1$. (b) The acyclic molecule $S(T-5)-1$.

Proof. If $n=2$ then $T$ is a path with two vertices. It is trivial to show that $S(T)-v$ contains a perfect matching. Hence we may assume that $n>2$ and proceed by induction on $n$. We distinguish the following two cases:

(i) $\quad v$ is a pendant vertex of $T$.

Since $T$ contains at least two pendant vertices, we may suppose that $u$ and $v$ are two pendant vertices of $T$. Let $e_{1}=(u, x)$ and $e_{2}=(v, y)$ be the two pendant edges of $T$. Hence, by induction, $S(T-u)-v$ contains a perfect matching, denoted by $M_{1}$. It is obvious that $S(T)-v$ contains a perfect matching $M_{1} \cup$ $\left\{\left(u, e_{1}\right)\right\}$. For the acyclic molecule $T$ illustrated in figure 1(a), vertices 1 and 5 are two pendant vertices, and $e_{1}=(1,2)$ and $e_{4}=(5,4)$ are two pendant edges of $T$. Note that $S(T-5)-1$ contains a perfect matching $M_{1}=\left\{\left(2, e_{1}\right),\left(3, e_{2}\right),\left(4, e_{3}\right)\right\}$ (see figure 2(b)). Hence $S(T)-1$ contains a perfect matching $M_{1} \cup\left\{\left(5, e_{4}\right)\right\}$ (see figure 2(a)).

(ii) $v$ is not a pendant vertex of $T$.

Suppose that $T-v$ has $k$ components $T_{1}, T_{2}, \ldots, T_{k}(k>1)$. Then $S(T)$ has $k$ components $S\left(T_{i}+v\right)-v$ for $1 \leqslant i \leqslant k$. By Case $(i)$, each $S\left(T_{i}+v\right)-v$ contains a perfect matching. Hence $S(T)-v$ contains a perfect matching.

Hence we have finished the proof of the lemma.

Theorem 3.2. Let $T$ be an acyclic molecule with $n$ vertices and $v$ an arbitrary vertex of $T$. Then

$$
W(T, v)=m(S(T)-v, n-2),
$$

where $W(T, v)$ is the partial Wiener index defined in (2).

Proof. If $n=2$, it is trivial to show that the theorem holds. Hence we may assume that $n \geqslant 3$ and proceed by induction on $n$. Without loss of generality, we may assume that the neighbors of $v$ in $T$ are $v_{1}, v_{2}, \ldots, v_{k}$ and $e_{1}=\left(v, v_{1}\right), e_{2}=$ $\left(v, v_{2}\right), \ldots, e_{k}=\left(v, v_{k}\right)$. Hence $T-v$ has $k$ components, denoted by $T_{1}, T_{2}, \ldots, T_{k}$, where $v_{i} \in V\left(T_{i}\right)$ for $i=1,2, \ldots, k$ (see figure 3(a)). Obviously, $S(T)$ has $k$ components $S\left(T_{1}\right)+e_{1}, S\left(T_{2}\right)+e_{2}, \ldots, S\left(T_{k}\right)+e_{k}$ (see figure 3(b)). 


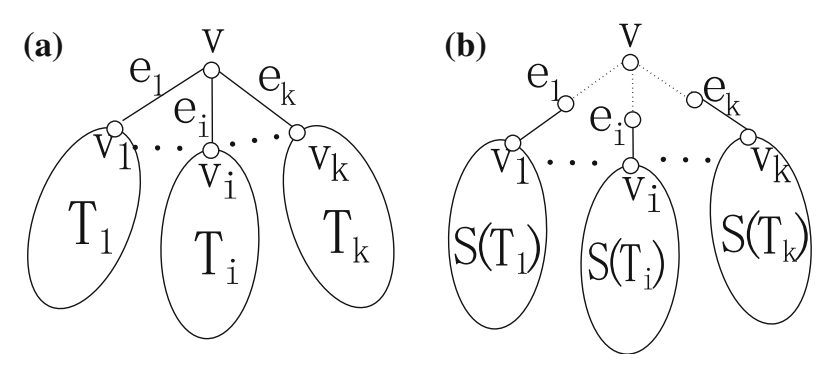

Figure 3. (a) The acyclic molecule $T$. (b) The acyclic graph $S(T)-v$.

By the definition of the partial Wiener index defined in (2), we have

$$
W(T, v)=\sum_{i=1}^{k} W\left(T_{i}+v, v\right),
$$

where $T_{i}+v=T-T_{1}-T_{2}-\cdots-T_{i-1}-T_{i+1}-\cdots-T_{k}$ for $i=1,2, \ldots, k$. Note that $S\left(T_{i}\right)+e_{i}=S\left(T_{i}+v\right)-v$. Hence, by lemma 3.1, $S\left(T_{i}\right)+e_{i}$ contains a perfect matching, which contains $\left|V\left(T_{i}\right)\right|$ edges. So we have

$$
m(S(T)-v, n-2)=\sum_{i=1}^{k} m\left(S\left(T_{i}\right)+e_{i},\left|V\left(T_{i}\right)\right|-1\right) .
$$

Since $v$ is a pendant vertex of $T_{i}+v$, for $1 \leqslant i \leqslant k$ we have

$$
W\left(T_{i}+v, v\right)=W\left(T_{i}, v_{i}\right)+\left|V\left(T_{i}\right)\right| .
$$

By induction, we have

$$
W\left(T_{i}, v_{i}\right)=m\left(S\left(T_{i}\right)-v_{i},\left|V\left(T_{i}\right)\right|-2\right) .
$$

Note that we have

$$
\begin{aligned}
& m\left(S\left(T_{i}\right)+e_{i},\left|V\left(T_{i}\right)\right|-1\right) \\
& \quad=m\left(S\left(T_{i}\right),\left|V\left(T_{i}\right)\right|-1\right)+m\left(S\left(T_{i}\right)-v_{i},\left|V\left(T_{i}\right)\right|-2\right) .
\end{aligned}
$$

By corollary 3.1, $m\left(S\left(T_{i}\right),\left|V\left(T_{i}\right)\right|-1\right)=\left|V\left(T_{i}\right)\right|$. Thus, by (19) $-(21)$, we obtain the following:

$$
\begin{aligned}
& m\left(S(T)+e_{i},\left|V\left(T_{i}\right)\right|-1\right)=\left|V\left(T_{i}\right)\right|+m\left(S\left(T_{i}\right)-v_{i},\left|V\left(T_{i}\right)\right|-2\right) \\
& \quad=\left|V\left(T_{i}\right)\right|+W\left(T_{i}, v_{i}\right)=W\left(T_{i}+v, v\right) .
\end{aligned}
$$

Hence (16) is immediate from (17), (18) and (22). The theorem thus follows. 


\section{The Wiener index of the acyclic molecule with a perfect matching}

In Section 3, we explained the Wiener index of an acyclic molecule $T$ with $n$ vertices as the number of matchings of $S(T)$ with $n-2$ edges. In this section, we show that if $T$ has a perfect matching then the Wiener index of $T$ can be expressed in terms of $m(S(T), i)$ for $1 \leqslant i \leqslant n-3$, that is, we have the following:

Theorem 4.3. Let $T$ be an acyclic molecule with $n \geqslant 4$ vertices, which contains a perfect matching. Then

$$
W(T)=\frac{1}{2} n+\sum_{i=0}^{n-3} m(S(T), i) 2^{n-i-2}
$$

Proof. Since $T$ has a perfect matching, $T$ must has the form showed in figure 4(a), where $T_{1}$ is an acyclic molecule with $n-2$ vertices, which contains a perfect matching. Hence $S(T)$ has the form illustrated in figure 4(b). Note that the characteristic polynomial $\phi(S(T), x)$ of $S(T)$ satisfies the following:

$$
\phi(S(T), x)=\phi\left(S\left(T_{1}\right), x\right)\left(x^{4}-3 x^{2}+1\right)-\phi\left(S\left(T_{1}\right)-v, x\right)\left(x^{3}-2 x\right),
$$

where $v$ is illustrated in figure 4(b). Hence we have

$$
\phi(S(T), \sqrt{2})=-\phi\left(S\left(T_{1}\right), \sqrt{2}\right) .
$$

By induction on $n$, from (24) we can prove the following:

$$
\phi(S(T), \sqrt{2})=0 .
$$

By (14) and (25), we have

$$
\sum_{k=0}^{n-1} m(S(T), k) 2^{n-1-k}=0 .
$$

Note that, by theorem 3.1 and corollary 3.1, we have $m(S(T), n-1)=n$ and $m(S(T), n-2)=W(T)$. Hence (23) is immediate from (26). Thus the theorem has been proved.

Remark 4.1. The above result implies that if $T$ is an acyclic molecule with $n$ vertices and with a perfect matching then $W(T)$ is even if and only if $n=0(\bmod 4)$. This result was previously found in [29-31]. 


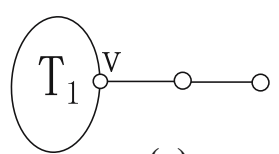

(a)

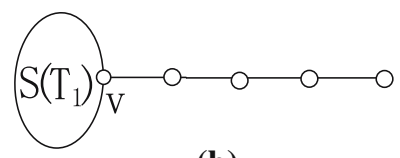

(b)

Figure 4. (a) The acyclic molecule $T$. (b) The acyclic molecule $S(T)$.

From the proof of theorem 4.3, we know that $\phi(S(T), \sqrt{2})=0$. By (11), we have $u(T, 2)=0$, which implies the following:

Corollary 4.3 (Guo and Tan [32). If $T$ is an acyclic molecule with a perfect matching. Then 2 is a root of the Laplacian characteristic polynomial of $T$.

\section{References}

[1] M. Randić and J. Zupan, J. Chem. Inf. Comput. Sci. 41 (2001) 550-560.

[2] D. J. Klein, D. Mihalić, D. Plavšić and N. Trinajstić, J. Chem. Inf. Comput. Sci. 32 (1992) 304-305.

[3] I. Gutman, J. Chem. Inf. Comput. Sci. 34 (1994) 1087-1089.

[4] H. Wiener, J. Am. Chem. Soc. 69 (1947) 17-20.

[5] H. Wiener, J. Am. Chem. Soc. 69 (1947) 2636-2638.

[6] H. Wiener, J. Phys. Chem. 52 (1948) 425-430.

[7] H. Wiener, J. Phys. Chem. 52 (1948) 1082-1089.

[8] H. Wiener, J. Chem. Phys. 15 (1947) 766-766.

[9] H. Hosoya, Bull. Chem. Soc. Jpn. 4 (1971) 2332-2339.

[10] B.Y. Yang and Y.N. Yeh, Adv. Appl. Math. 16 (1995) 72-94.

[11] B.Y. Yang and Y.N. Yeh, In: Proc. of Second International Taiwan-Moscow Algebra Workshop (Tainan, 1997), eds. Y. Fong et al. (Springer, Hong Kong, 2000) pp. 203-226.

[12] W.C. Huang, B.Y. Yang and Y.N. Yeh, Discrete Appl. Math. 73 (1997) 113-131.

[13] I. Gutman, J. Serb. Chem. Soc. 68 (2003) 949-952.

[14] M. Randić, Chem. Phy. Lett. 211 (1993) 478-483.

[15] R. Merris, Multlinear A. 25 (1989) 291-296.

[16] R. Merris, J. Graph Theory. 14 (1990) 365-369.

[17] B. Mohar, The Laplacian spectrum of graphs, in: Graph Theory, Combinatorics, and Applications, eds. Y. Alavi, G. Charchand, O.R. Ollermann and A.J. Schwenk, (Wiley, New York, 1991), pp. 871-898.

[18] B. Mohar, Graphs Combin. 7 (1991) 53-64.

[19] I. Gutman, S.L. Lee, C.H. Chu and Y.L. Luo, Indian J. Chem. 33A (1994) 603-608.

[20] B. Mohar, D. Babić and Trinajstić, J. Chem. Inf. Comput. Sci. 33 (1993) 153-154.

[21] O. Chan, T.K. Lam and R. Merris, J. Chem. Inf. Comput. 37 (1997) 762-765.

[22] O. Chan, I. Gutman, T.K. Lam and R. Merris, J. Chem. Inf. Comput. Sci. 38 (1998) 62-65.

[23] J.K. Doyle and J.E. Graver, Discrete Math. 7 (1977) 147-154.

[24] E.R. Canfield, R.W. Robinson and D.H. Rouvray, J. Comput. Chem. 6 (1985) 598-609.

[25] A.A. Dbrumom and I. Gutman, Publ. Inst. Math. (Beograd). 56 (1994) 18-21.

[26] I. Gutman, Y.N. Yeh, S.L. Lee and J.C. Chen, MATCH Commun. Math. Comput. Chem. 30 (1994) 103-115. 
[27] D.M. Cvetkocić, M. Doob and H. Sachs, Spectra of Graphs-Theory and Application (Academic Press, New York, 1980).

[28] I. Gutman, D. Vidović and B. Furtula, Indian J. Chem. 42A (2003) 1272-1278.

[29] I. Gutman. J. Chem. Inf. Comput. Sci. 34 (1994) 912-914.

[30] I. Lukovits. J. Chem. Inf. Comput. Sci. 33 (1993) 626-629.

[31] A.A. Dobrynin, R. Entringer and I. Gutman, Acta Appl. Math. 66 (2001) 211-249.

[32] J. Guo and S. Tan, Linear Alg. Appl. 325 (2001) 71-74. 